\title{
Towards an Adaptive Upper Limb Rehabilitation Game with Tangible Robots
}

\author{
Arzu Guneysu Ozgur ${ }^{1}$, Louis P. Faucon ${ }^{1}$, Pablo Maceira-Elvira ${ }^{2}$, Maximilian J. Wessel ${ }^{2}$, Wafa Johal ${ }^{1,4}$, \\ Ayberk Özgür, Andéol Cadic-Melchior ${ }^{2}$, Friedhelm C. Hummel ${ }^{2,3, *}$ and Pierre Dillenbourg ${ }^{1, *}$
}

\begin{abstract}
A key feature of a successful game is its ability to provide the player with an adequate level of challenge. However, the objective of difficulty adaptation in serious games is not only to maintain the player's motivation by challenging, but also to ensure the completion of training objectives.

This paper describes our proposed upper-limb rehabilitation game with tangible robots and investigates the effect of game elements and gameplay on the amount of the performed motion in several planes and percentage of failure by using the data from 33 unimpaired subjects who played 53 games within two consecutive days. In order to provide a more generic adaptation strategy in the future, we discretize the game area to circular zones. We then show the effect of changing these zones during gameplay on the activation of different muscles through EMG data in a pilot study.

The study shows that it is possible to increase the challenge level by adding more active agents chasing the player and increasing the speed of these agents. However, only the increase in number of agents significantly increases the users' motion on both planes. Analysis of player behaviors leads us to suggest that by adapting the behaviour of these active agents in specific zones, it is possible to change the trajectory of the user, and to provide a focus on the activation of specific muscles.
\end{abstract}

\section{INTRODUCTION}

Previous studies have shown that effective rehabilitation depends on the quality and the amount of physical activities, the patients' active participation, and commitment to the therapeutic session [1], [2], [3]. In order to improve engagement and increase the intensity of exercise, new rehabilitation technologies commonly include game-like elements such as entertaining graphics, automated difficulty adaptation, and feedback mechanism [2], [4], [5], [6], [7], [8], [9], [10], [11]. These games typically also help therapists support patient motivation and measures their progression.

Apart from these game features, the adaptation of a serious game to its application context also plays a crucial role in ensuring the game's acceptability by therapists as well as the users. Since patients who need upper limb rehabilitation generally have limited physical abilities, the difficulty of a task can be defined by the amount of physical effort

\footnotetext{
This work is supported by NCCR Robotics.

${ }^{1}$ Arzu Guneysu Ozgur, Pierre Dillenbourg, Jennifer K. Olsen, Wafa Johal and Louis P. Faucon are with CHILI Lab, EPFL, Switzerland

${ }^{2}$ Maximilian J. Wessel, Pablo Maceira-Elvira, Andéol Cadic-Melchior and Friedhelm C. Hummel are with, UPHUMMEL, Center for Neuroprosthetics, Brain Mind Institute, Defitech Chair of Clinical Neuroengineering, EPFL, EPFL Valais and Clinique Romande de Réadaptation, Switzerland.

${ }^{3}$ Friedhelm C. Hummel is with Clinical Neuroscience, University of Geneva Medical School, Switzerland.

${ }^{4}$ Wafa Johal is with the LSRO Lab at EPFL, Switzerland.

${ }^{*}$ Both authors contributed equally.
}

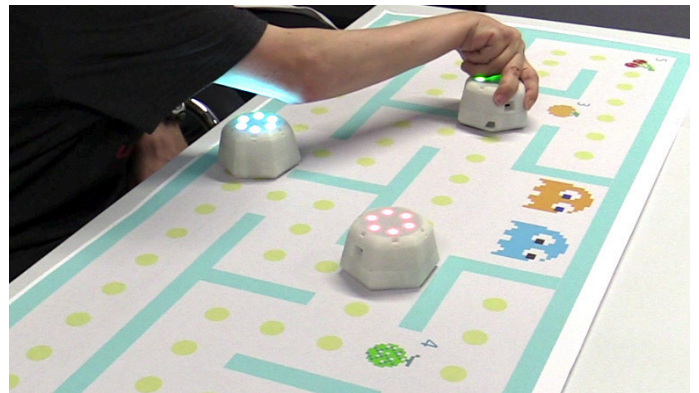

Fig. 1: Pacman activity with Cellulo [13].

necessary to perform it. It should naturally be adapted to the abilities of the patient, as well as targeted exercise activities. This means it should also challenge the user to improve training outcomes.

Most difficulty adaptation techniques in upper-limb rehabilitation games focus on the maximization of effort during the rehabilitation session [12]. Adaptation strategies often adjust the number of targets, speed of targets and range of motion according to the movement amplitude, accuracy and velocity of the motion, typically without focusing on the activation of target muscle groups and on the game elements that may affect these.

The aim of this study is to further evaluate the effect of game elements of our previously developed rehabilitation game platform on the rate of failure (which is one dimension of the challenge) and the effect on different motion types which are the expected exercises in an effective rehabilitation session that maximizes effort. The study explores user motion performances using the data from 33 unimpaired subjects who played 53 games within two consecutive days.

In addition, we provide the proof of concept of a discretized 2D game area with circular zones and its relation to the activation of different muscles, through EMG data collected in a pilot experiment. We conclude with a discussion on how user motion can be manipulated through game elements to provide targeted muscle activation, which will enable adaptive game design in the future.

\section{REHABILITATION SYSTEM OVERVIEW}

Our gamified rehabilitation platform consists of tangible Cellulo robots and a range of mazes printed on paper sheets designed with a theme close to the Pacman game [13]. Cellulo is a handheld, small-sized, mobile, haptic robot that operates on such printed paper sheets. These graspable robots 
can be used as an interface for interacting with many virtual objects that reside on a plane [14].

The robots are designed to be simple to operate; all robots are connected wirelessly to a mobile device (a tablet or smartphone) that runs the activity and game logic. The system includes self-localization on the activity sheet covered with a dot pattern [15]; holonomic motion robust against human manipulation [16]; six touch buttons (independently back-illuminated in full RGB) and wireless Bluetooth communication [17]. The platform provides fast $(>90 \mathrm{~Hz})$ and accurate localization (sub-mm) of many robots which can be logged to record all the interactions during the game, such as user motion.

The activity itself is printed on paper sheets that can feature any desired graphical game elements defined as active zones. These zones can be associated with specific robot behaviors to design game logic (e.g. in Fig. 1 blue walls activate assistive haptic behaviour of the robot, while fruits represent target objects to be collected in a game). An activity is therefore the combination of the paper elements, the robots with particular interaction modalities and the tablet that runs the activity-specific software. As such, the role of the robots and paper depends on the design of each particular activity.

The Cellulo for rehabilitation project aims to provide practical, easy to use and intuitive gamified upper-arm rehabilitation by using these tangible robots as game agents and objects. Our first game, Pacman, is designed iteratively with participation of stroke, brachial plexus and cerebral palsy patients (18 in total) and seven therapists in four different therapy centers [13].

There are six targets in the game that have been gamified as apples for the Pacman to eat. The user is expected to collect all six apples as quickly and precisely as possible to finish the game (we define precision as not crashing into the maze walls). The active agents (called ghosts) chase the user's robot during the game in order to catch it; all previously collected apples are lost and the ghosts return to their initial positions if this happens. The game then restarts from the beginning until all six apples are eventually collected. The players are informed that the goal is to collect all apples as quickly as possible and are informed of their score (total time) at the end of each game in order to increase their motivation.

Several game elements are designed for tuning speed, accuracy, range of motion and challenge of the game play. These game elements are:

- A range of maps with different mazes and sizes

- One vs. two ghost robot(s) chasing the Pacman

- Speed of the chasing robot(s)

- Optional rules:

- Turn rule: User can only collect the fruits by rotating the robot on top of them

- Cross border penalty rule: User loses the last eaten fruit when he/she crashes into a wall

- Haptic feedback rule: Robot provides informative haptic assistance when the user crashes into a wall

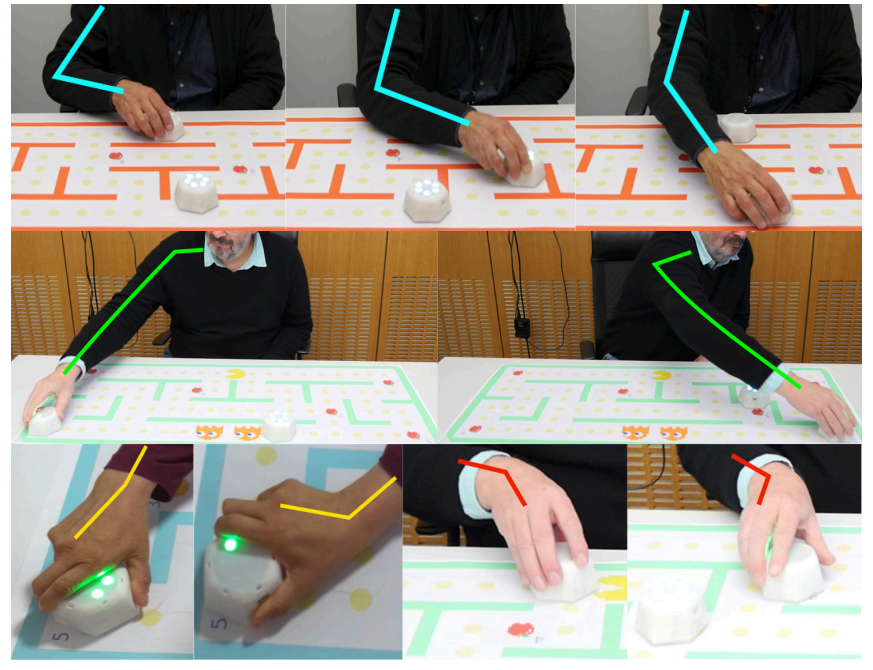

Fig. 2: Expected exercise activities during game motions.

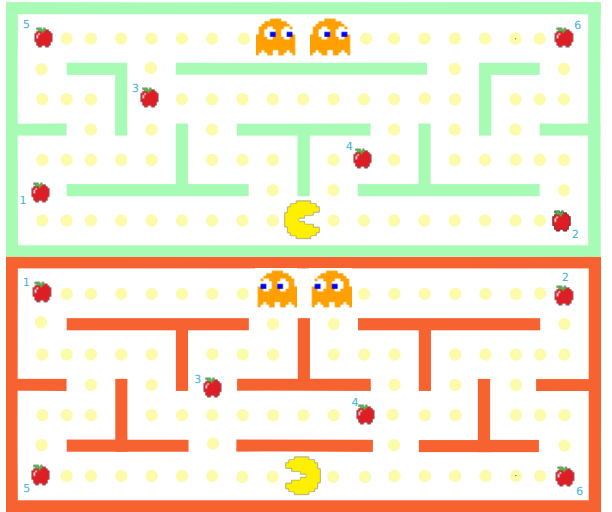

Fig. 3: Maps used during the experiments (420x960 mm).

\section{MAPPING OF GAME MOTIONS TO EXERCISE ACTIVITIES}

The crucial feature of a rehabilitation game is to include the appropriate exercise motions. Naturally, since the basis of our games is tabletop robots, we focus on upper limb activities where the tangible robots serve as end-effectors for the patients' upper limbs. Although the robots perform movements in the transversal plane (on the paper), joints of the limb holding the robot may still move in a threedimensional space.

The maps are designed according to the possible range of motion of the human, and the following basic motions commonly used in stroke rehabilitation exercises are expected during the given corresponding actions:

- Moving the robot along the vertical axis of the paper: Elbow flexion and extension (see Fig. 2 top)

- Moving the robot along the horizontal axis of the paper: Shoulder abduction and adduction (see Fig. 2 middle)

- Rotating the robot or manipulating the robot through corners: Grasping and wrist ulnar and radial deviation (see Fig. 2 bottom)

\section{DATA COLLECTION}

In order to have a baseline motion trajectory pool to use for comparison purposes, we collected data from 33 
unimpaired participants aged between 18 and 80 . The inclusion criteria were as follows: Right-handedness, normal values of Mini-mental state examination ( $>26 / 30$ ), and $\geq 18$ years old. Exclusion criteria were: Neuropsychiatric diseases, history of seizures, musculoskeletal dysfunction that compromises finger movement, professional musicians or intense professional usage of a computer keyboard. The study has been conducted in accordance with the World Medical Association's Declaration of Helsinki. The study protocol was approved by the cantonal ethics committee of Vaud, Switzerland (project number 2017-00765). Approval and informed written consent was obtained from all subjects prior to the inclusion in the study.

All participants took part in two sessions in our laboratory at EPFL Lausanne Campus or EPFL Campus Biotech in Geneva. Each participant played 53 games on two different maps (see Fig. 3) within two consecutive days of experiments with changing game configurations and increasing difficulty per map.

Since the data collection is a baseline for comparison purposes, we started from the easiest game configuration and progressively increased the difficulty by introducing a new game element one at a time: We start with $20 \mathrm{~mm} / \mathrm{s}$ ghost speed for the very first game of each day for each user, which we then set to $40 \mathrm{~mm} / \mathrm{s}, 60 \mathrm{~mm} / \mathrm{s}$ and $60 \mathrm{~mm} / \mathrm{s}$ for the next three games. Next, the assistive haptic feedback is enabled and two more games with $60 \mathrm{~mm} / \mathrm{s}$ ghost speed are played. Following that, the cross border penalty rule is enabled and two more games are played with $60 \mathrm{~mm} / \mathrm{s}$ and $100 \mathrm{~mm} / \mathrm{s}$ ghost speed. Next, the turn rule is introduced and two more games are played with $60 \mathrm{~mm} / \mathrm{s}$ and $100 \mathrm{~mm} / \mathrm{s}$ ghost speed. Finally, a second ghost is introduced and three more games are played with $60 \mathrm{~mm} / \mathrm{s}, 100 \mathrm{~mm} / \mathrm{s}$ and 100 $\mathrm{mm} / \mathrm{s}$ ghost speed, which marks the end of the games (total of 13) played with the first map.

For the second map, all above configurations except the first two (with $20 \mathrm{~mm} / \mathrm{s}$ and $40 \mathrm{~mm} / \mathrm{s}$ ghost speed and no rules) are repeated. After this repetition, two extra games are played with the hardest configuration which includes the cross border penalty rule, turn rule, $100 \mathrm{~mm} / \mathrm{s}$ ghost speed and two ghosts (again, a total of 13 games), which marks the end of the first day. In the second day, the aforementioned games are repeated ${ }^{1}$.

During the game play, the position $(x, y)$ and the orientation $(\theta)$ of each robot are recorded with around 93 $\mathrm{Hz}$ frequency and sub-mm accuracy, including the robot controlled by the user. All events and interactions within the game (e.g fruit collection, kidnapping of the robot and wall crashes) are also recorded with their timestamps.

\section{METHODOLOGY}

\section{A. Map Discretization with Circular Zones}

In order to provide a mapping between the game motions and expected exercise activities, a more generic representa-

\footnotetext{
${ }^{1}$ For the first day only, a 27 th game is played with an easy configuration for the purposes of measuring any overnight learning effect, whose results are not discussed in this paper.
}

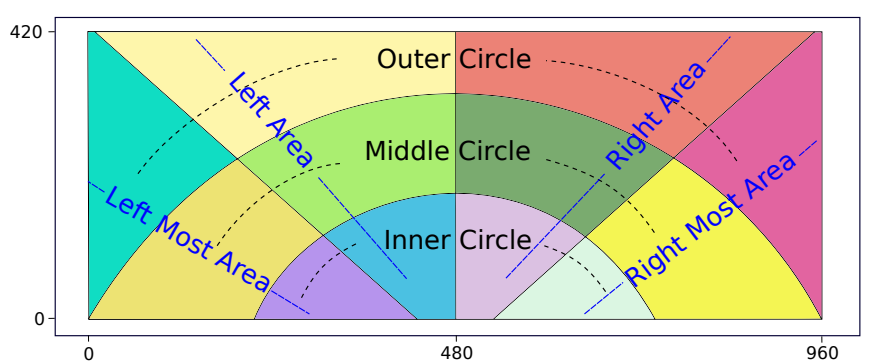

Fig. 4: Map discretization.

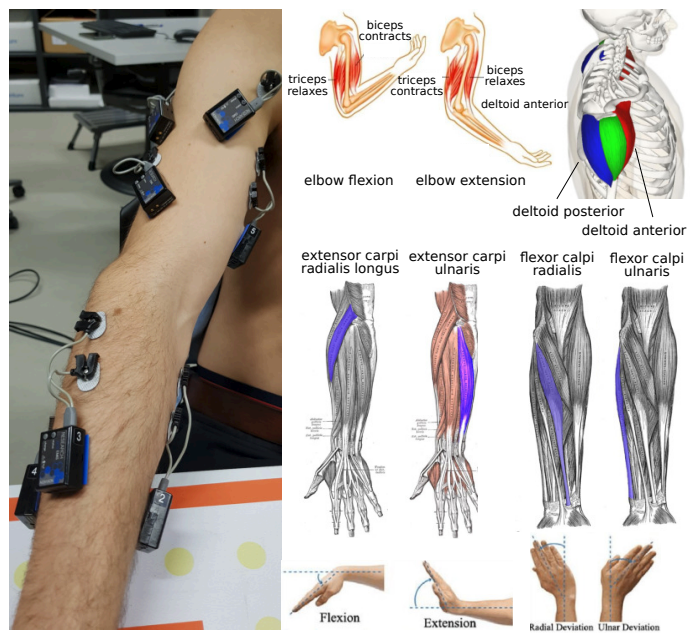

Fig. 5: The 8 muscles related to our expected exercise motions and EMG positioning on the subject's arm.

tion is defined to apply to any map that may have similar range of motions. Since the human shoulder activity is circular in nature, the area is divided into circular "ring" zones by placing the user at the middle of the approximate circle where he/she is typically seated. These zones are each further divided into 4 axial regions from left to right; see Fig. 4 for a detailed visualization. With this division, moving left/right through circular areas are mainly expected to correspond to shoulder abduction/adduction and moving between circles backwards and forwards are mostly expected to correspond to elbow extension/flexion.

\section{B. Muscle Activation with Zone Transitions}

In order to test the proposed discretization, the effect of gameplay on specific muscles related to the expected motions is investigated apart from the main study in the form of a pilot test. Data from two healthy adults (mean age $=26$ ) are collected while they played 6 games with 3 different configurations: One ghost with $100 \mathrm{~mm} / \mathrm{s}$ speed, two ghosts with $100 \mathrm{~mm} / \mathrm{s}$ speed and two ghosts with $100 \mathrm{~mm} / \mathrm{s}$ speed with turn rule.

Muscle activity was captured using eight Electromyography (EMG) sensors placed on muscles thought to play main roles in movements performed during the game: Triceps, biceps, deltoid anterior, deltoid posterior, extensor carpi radialis longus, extensor carpi ulnaris, flexor calpi radialis and flexor calpi ulnaris (Fig. 5). EMG was recorded using a Noraxon DTS Receiver (https://www.noraxon.com/), with a sampling frequency of $3 \mathrm{kHz}$, high-pass filter of $10 \mathrm{~Hz}$ 


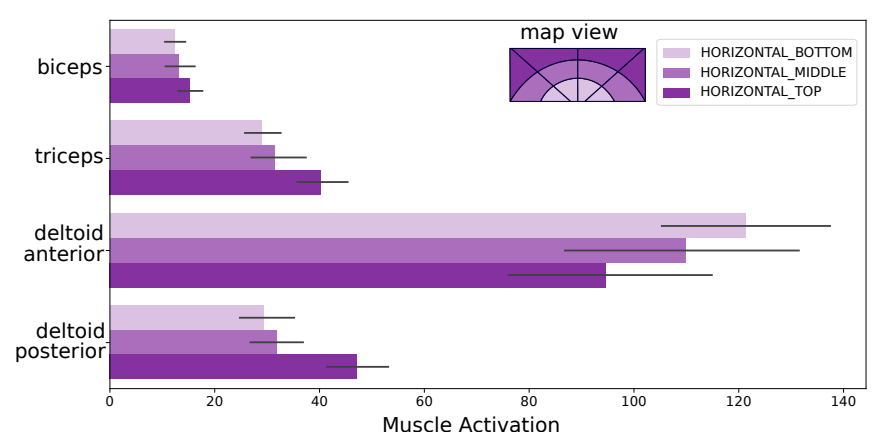

Fig. 6: Muscle activation while moving axially within the same circles.

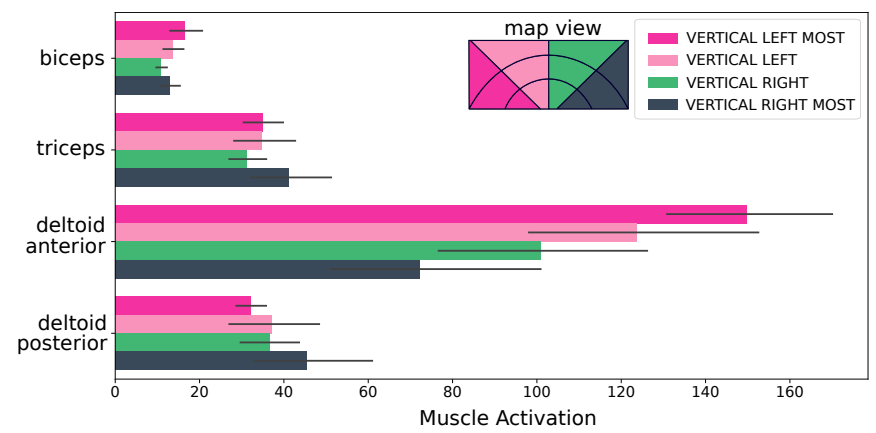

Fig. 7: Muscle activation while moving forward-backward within the same axial areas.

and low-pass filter of $1 \mathrm{kHz}$. The activity in the four main muscles involved in the navigation of the map, namely those of the shoulder (deltoid) and the upper arm (biceps and triceps) are depicted in Fig. 6 through Fig. 9 under different zone transition and motion conditions. Activity was characterized using the root mean square (RMS), as it correlates directly to applied force [18].

Navigation of the map requires the orchestrated activation of several muscles depending on the posture held and the direction of movement. Holding the robot as done by the participant shown in Fig. 2 requires a continuous contraction of deltoid muscles (mainly anterior, but complemented with lateral and posterior segments for stability), as well as continuous contraction of biceps and triceps to stabilize the elbow (to a lesser extent, as they do not carry the weight of the upper arm). Transversal displacements over the map require additional contraction of these same muscles, depending on the direction of movement (muscles only pull, i.e. displacement to the left will require additional contraction of deltoid anterior and biceps muscles). This description can be observed in Fig. 6 to Fig. 9 where all figures show larger activation of the deltoid anterior muscle compared to other muscles, as it is carrying the weight of the entire arm due to the posture held. Further analysis relating muscle activation to posture (i.e. biomechanical analysis) is necessary to better understand the ratio of activation between i.e. biceps and triceps, which will be done in the future. Fig. 8 and Fig. 9 both show expected increase in muscle output for antagonist muscles (muscles pulling in the direction of movement).

Activity on wrist flexor and extensor muscles (Fig. 10) was most apparent when participant forced the robot to turn on

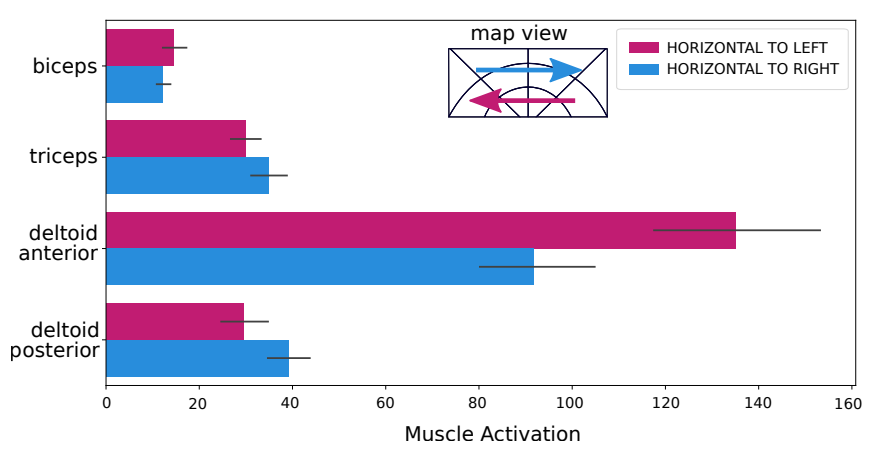

Fig. 8: Muscle activation while moving horizontally, specific to direction (without grouping zones).

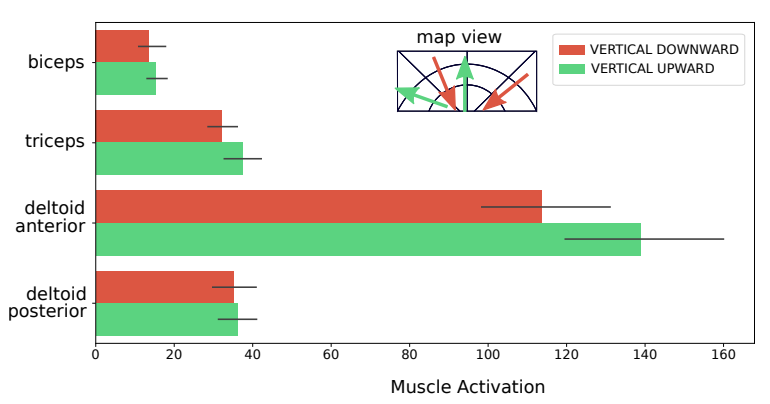

Fig. 9: Muscle activation while moving forward-backward, specific to direction (without grouping zones).

its own axis i.e. under the presence of the turn rule. Further study of the relationship between torsion angle and muscle activity is needed to adequately adjust training in patients.

Understanding of these patterns of activation is crucial for an eventual optimization of rehabilitation in patients. This observed activity, such as increased output in deltoid anterior, might not be present in patients, as they are frequently unable to hold their arms up and place higher pressure on the robots. Further analyses will need to account for orchestrated activation of muscles (i.e. muscle synergies), which might be used for therapeutic planning in patients seeking to promote specific synergistic patterns [19]. Additionally, adjustments to certain requirements (i.e. robot rotation angle) would be necessary in patients with limited range of motion at the wrist so as to keep training exercises within their present capabilities and avoid frustration during training.

Additional limitations to this initial analysis reside in measured muscles. Construction of a more comprehensive biomechanical model requires the inclusion of other muscles such as the pectoralis major, rhomboideus and trapezius (i.e.

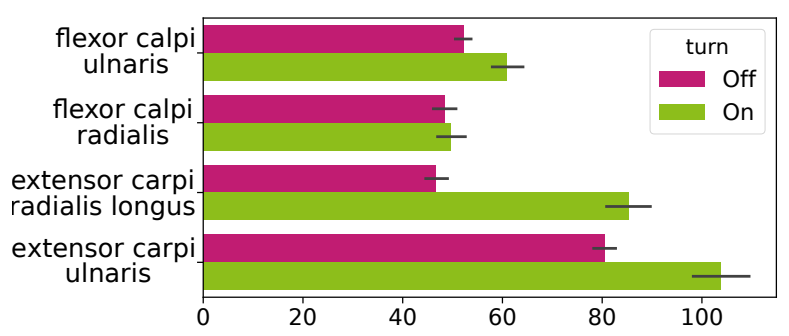

Fig. 10: Effect of turn rule to wrist muscle activation. 

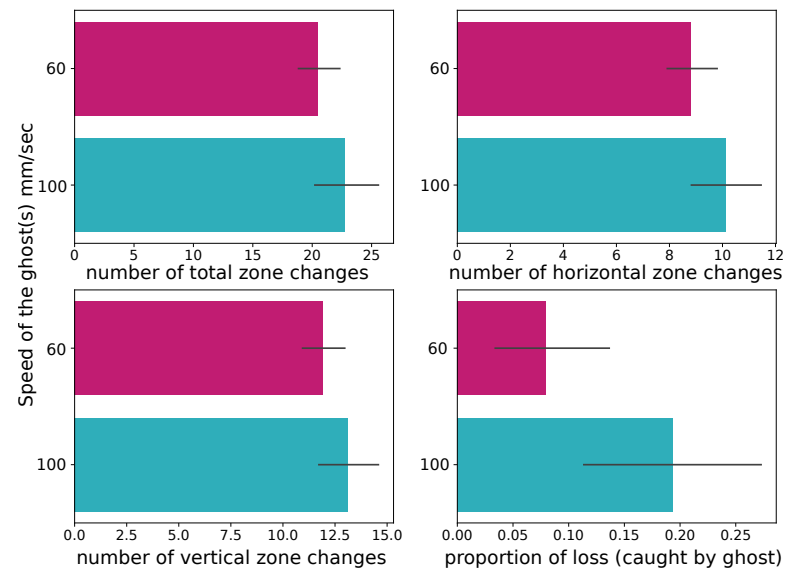

Fig. 11: Effect of speed on loss rate and motion.

to characterize vertical movements), as well as compensation done by the thorax. Further, other EMG features commonly used in literature [20] can be used to study more subtle differences in muscle activity between healthy subjects and patients.

\section{RESULTS}

\section{A. Effect of Game Elements on Frustration and Motion}

As previously mentioned, a number of game elements can be configured in order to ultimately make the game more challenging without making it frustrating or unbeatable. While the total amount of motion can be defined relatively straightforwardly as the number of zone changes in our game, defining frustration is more challenging as there is no loss condition. The game restarts upon the ghost(s) catching the user and finishes until all fruits are collected in one round. For this reason, we defined frustration as the rate in which users failed and restarted a game (over all games).

Fig. 11 shows the effects of two different ghost chasing speeds on the failure rate and the number of zone changes (which is a proxy for the total motion of the user). We found no significant difference $(p>0.05)$ for the number of zone changes but the failure rate was found to be significantly higher $(p<0.05)$. In Fig. 12 the effect of one vs. two ghosts chasing the user on the same measures are given, which show that adding an additional ghost to the game significantly increases the failure rate $(p<0.001)$. Furthermore, the additional ghost also increases the total motion of the user $(p<0.001)$ in both horizontal $(p<0.0001)$ and vertical directions $(p<0.001)$. Therefore, the additional active agent chasing the user improves both challenge and the amount of motion.

\section{B. Choice of Trajectory Direction Based on Closest Ghost}

The second investigation on data concerns the choice of trajectory of players based on position of the ghosts. For this, the angle between the chosen trajectory of the player and the direction of the closest ghost over a $250 \mathrm{~ms}$ time window is calculated. The histogram in Fig. 13 shows the flight angles of the players compared to ghost position. A Kolmogorov-Smirnov test shows that the chosen trajectory

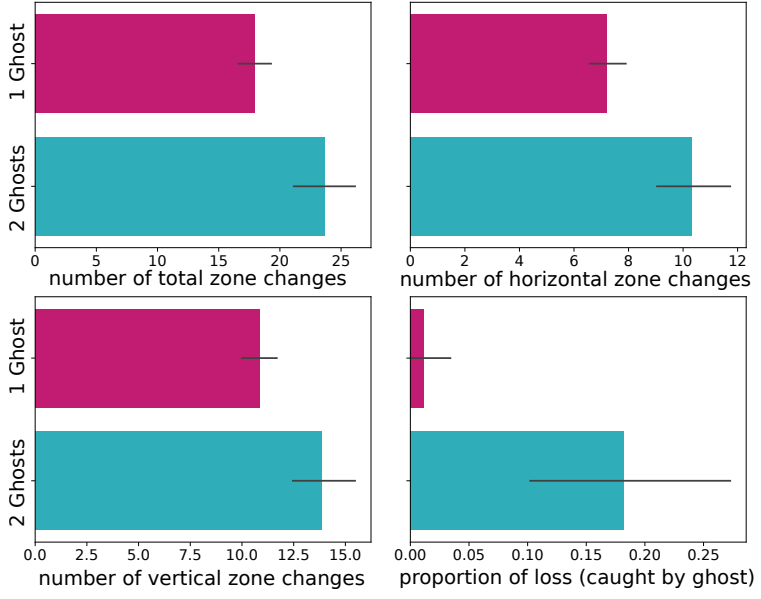

Fig. 12: Effect of ghost number on loss rate and motion.

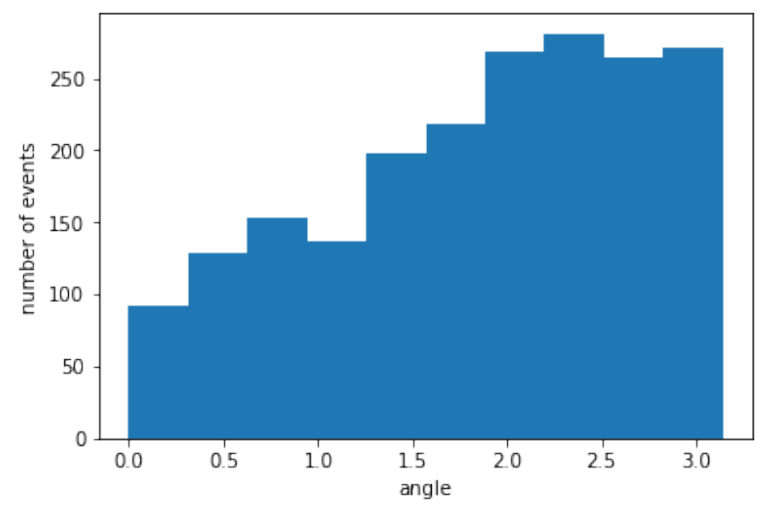

Fig. 13: Distribution of player trajectory angle (radian) with direction of the closest ghost - $\pi$ means that the player flees from the ghost, 0 means that the player approaches the ghost.

angle is significantly different from the uniform distribution $(p<0.0001)$.

\section{Density of Motions}

In order to visualize the user's motion trajectories, a heatmap representation is extracted over the data. Since the turn rule is expected to create a bias towards target points (i.e fruits), analysis is done by separating the games played when the turn rule is on and the games played when the turn rule is off. Looking at the results obtained for each map, the most visited areas during turn condition are targets since the user spends more time on those areas to collect the apples by rotating the Pacman while staying on the target (see top heatmaps in Fig. 14).

On the other hand, the most visited areas of the games not having turn rule are the targets on the corners, as well as the inner corners on the right and left most parts of the maps. Interestingly, corners closer to targets that do not reside on the corners of the maze seem to be visited more than these targets themselves (see bottom heatmaps in Fig. 14). These findings may indicate that adaptive strategies of the ghosts can also be tuned by the blocking apples and corners of the maze that will lead the user to move to the other corners and targets. 

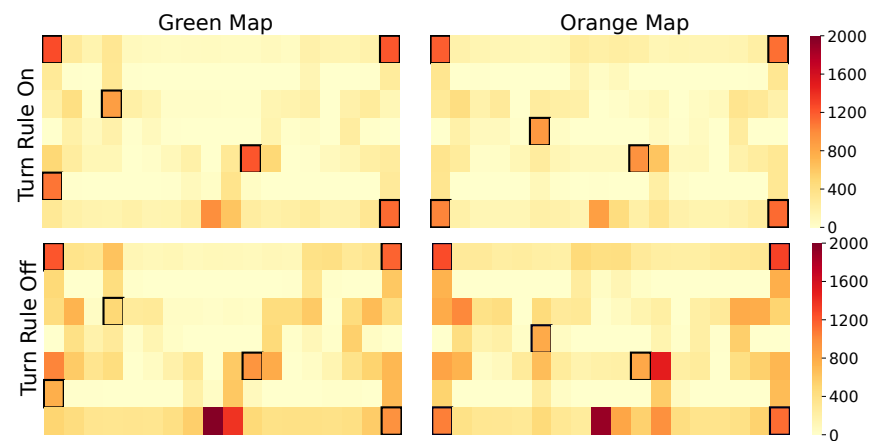

Fig. 14: Heatmaps representing the most visited areas.

\section{DISCUSSION AND FUTURE WORK}

In this paper, we showed that the game elements within our tabletop game with tangible robots can influence the amount and nature of player motion. Given the results here, these game elements can be appropriately selected by therapists to adapt the game to each patient's particular needs. Beyond the configuration capability, our analysis of player behavior suggests possibilities for other kinds of online adaptation. The fact that players often use the same paths on a particular map and that they tend to consistently avoid the ghosts shows that placing the ghosts to block specific areas of the map could lead the player to execute different motions targeting different muscles' activation. We can thus design adaptive ghost behaviors which would increase the quantity of a given type of motion that the game allows.

These findings rely on data collected from healthy individuals, for the purpose of constituting a baseline for future measurements. However, our future goal is to extend our methodology to patients (who require upper limb rehabilitation e.g stroke survivors) and their therapy sessions. Therefore, our immediate concern is to implement online adaptive strategies (through e.g ghost behaviors) and evaluate the effect of this adaptation on the training outcomes of patients during long term therapeutic sessions, especially when compared to other traditional games or methods. Particular challenges here include EMG signal variability in such patients when physically interacting with the robots, since each patient compensates differently for their specific impairments.

In this study, by discretizing the maze and ignoring the specific topology, we mainly focused on muscular aspects than cognitive aspects of the game. In future studies we will also investigate the cognitive part by focusing on the user's game-playing strategies.

\section{REFERENCES}

[1] G. Kwakkel, R. C. Wagenaar, T. W. Koelman, G. J. Lankhorst, and J. C. Koetsier. Effects of intensity of rehabilitation after stroke: a research synthesis. Stroke, 28(8):1550-1556, 1997.

[2] M. K. Holden and T. Dyar. Virtual environment training-a new tool for neurorehabilitation? Neurology Report, 26(2):62-71, 2002.

[4] R. Colombo, F. Pisano, A. Mazzone, C. Delconte, S. Micera, M. C. Carrozza, P. Dario, and G. Minuco. Design strategies to improve patient motivation during robot-aided rehabilitation. Journal of neuroengineering and rehabilitation, 4(1):3, 2007.

[3] M. C. Cirstea and M. F. Levin. Improvement of arm movement patterns and endpoint control depends on type of feedback during practice in stroke survivors. Neurorehabilitation and neural repair, 21(5):398-411, 2007.
[5] S. M. Nijenhuis, G. B. Prange, F. Amirabdollahian, P. Sale, F. Infarinato, N. Nasr, G. Mountain, H. J. Hermens, A. H. A. Stienen, J. H. Buurke, and J. S. Rietman. Feasibility study into self-administered training at home using an arm and hand device with motivational gaming environment in chronic stroke. Journal of neuroengineering and rehabilitation, 12(1):89, 2015.

[6] M. Goršič, M. H. Tran, and D. Novak. Cooperative cooking: A novel virtual environment for upper limb rehabilitation. In 2018 40th Annual International Conference of the IEEE Engineering in Medicine and Biology Society (EMBC), pages 3602-3605. IEEE, 2018.

[7] M. Pirovano, R. Mainetti, G. Baud-Bovy, P. L. Lanzi, and N. A. Borghese. Self-adaptive games for rehabilitation at home. In Computational Intelligence and Games (CIG), 2012 IEEE Conference on, pages 179186. IEEE, 2012.

[8] B. Rabin, G. Burdea, J. Hundal, D. Roll, and F. Damiani. Integrative motor, emotive and cognitive therapy for elderly patients chronic poststroke a feasibility study of the brightarm ${ }^{\mathrm{TM}}$ rehabilitation system. In Virtual Rehabilitation (ICVR), 2011 International Conference on, pages 1-8. IEEE, 2011.

[9] M. S. Cameirão, S. B. i Badia, Esther D. Oller, and P. F. M. J. Verschure. Neurorehabilitation using the virtual reality based rehabilitation gaming system: methodology, design, psychometrics, usability and validation. Journal of neuroengineering and rehabilitation, 7(1):48, 2010.

[10] M. Annett, F. Anderson, D. Goertzen, J. Halton, Q. Ranson, W. F. Bischof, and P. Boulanger. Using a multi-touch tabletop for upper extremity motor rehabilitation. In Proceedings of the 21st Annual Conference of the Australian Computer-Human Interaction Special Interest Group: Design: Open 24/7, pages 261-264. ACM, 2009.

[11] M. Mihelj, D. Novak, M. Milavec, J. Ziherl, A. Olenšek, and M. Munih. Virtual rehabilitation environment using principles of intrinsic motivation and game design. Presence: Teleoperators and Virtual Environments, 21(1):1-15, 2012.

[12] N. Hocine, A. Gouaïch, S. A. Cerri, D. Mottet, J. Froger, and I. Laffont. Adaptation in serious games for upper-limb rehabilitation: an approach to improve training outcomes. User Modeling and UserAdapted Interaction, 25(1):65-98, 2015.

[13] A. Guneysu Ozgur, M. J. Wessel, W. Johal, K. Sharma, A. Özgür, P. Vuadens, F. Mondada, F. C. Hummel, and P. Dillenbourg. Iterative design of an upper limb rehabilitation game with tangible robots. In Proceedings of the 2018 ACM/IEEE International Conference on Human-Robot Interaction, pages 241-250. ACM, 2018.

[14] A. Özgür, W. Johal, F. Mondada, and P. Dillenbourg. Haptic-enabled handheld mobile robots: Design and analysis. In Proceedings of the 2017 CHI Conference on Human Factors in Computing Systems, pages 2449-2461. ACM, 2017.

[15] L. Hostettler, A. Özgür, S. Lemaignan, P. Dillenbourg, and F. Mondada. Real-time high-accuracy $2 \mathrm{~d}$ localization with structured patterns. In Robotics and Automation (ICRA), 2016 IEEE International Conference on, pages 4536-4543. IEEE, 2016.

[16] A. Özgür, W. Johal, and P. Dillenbourg. Permanent magnet-assisted omnidirectional ball drive. In Proceedings of the 2016 IEEE/RSJ International Conference on Intelligent Robots and Systems (IROS), number CONF. IEEE, 2016.

[17] A. Özgür, S. Lemaignan, W. Johal, M. Beltran, M. Briod, L. Pereyre, F. Mondada, and P. Dillenbourg. Cellulo: Versatile handheld robots for education. In Proceedings of the 2017 ACM/IEEE International Conference on Human-Robot Interaction, pages 119-127. ACM, 2017.

[18] A. Gijsberts, M. Atzori, C. Castellini, H. Muller, and B. Caputo. Movement error rate for evaluation of machine learning methods for semg-based hand movement classification. IEEE Trans. Neural Syst. Rehabil. Eng, 22(4):735-744, 2014.

[19] V. C. K. Cheung, A. Turolla, M. Agostini, S. Silvoni, C. Bennis, P. Kasi, S. Paganoni, P. Bonato, and E. Bizzi. Muscle synergy patterns as physiological markers of motor cortical damage. Proceedings of the National Academy of Sciences, 109(36):14652-14656, 2012.

[20] A. Phinyomark, P. Phukpattaranont, and C. Limsakul. Feature reduction and selection for emg signal classification. Expert Systems with Applications, 39(8):7420-7431, 2012. 